\title{
Hibernoma in the Thoracic Back Muscle Accompanied by Neurilemmoma
}

\author{
Hyun Su Kim, Sang Gu Lee, Seong Son, Keun Lee \\ Department of Neurosurgery, Gachon University Gil Medical Center, Incheon, Republic of Korea
}

Hibernoma is a very rare, benign soft fatty tumor that derived from remnants of fetal brown tissue. The following case of hibernoma break out occurred from a patient who had received surgical resection of neurilemmoma. There has never been a case that reported about hibernoma accompanied by neurilemmoma. It is anticipated that this experience would be helpful to other physicians who are interested in this rare type of tumor.

Key Words: Hibernoma $\cdot$ Atypical lipoma $\cdot$ Neurilemmoma

\section{INTRODUCTION}

Hibernoma is a very rare fatty tumor that originates from remaining brown fat after one's fetal stage. Fetal brown tissue is known to be found from pelvic limb, scapular region, axilla, collum, abdominal etc., and observed from a mass, gradually increasing in size. So far, there was hardly a case of a malignant tumor, and it is established as a ground rule to treat such conditions by a total resection of affected parts.

The following study reports about hibernoma that developed from a patient with a history of neurilemmoma and discussion of literature.

\section{CASE REPORT}

A 49-year-old man presented with a mass on his back which had been growing for several months. A year ago, he had his previous visit with symptoms of a stiff shoulder on his right scapular region that had last for a few months. At that time, he underwent chest X-ray and thoracic MRI and discovered a paraspinal mass on the right side of third thoracic vertebra. He had a resection (Fig. 1) after being diagnosed as neurilemmoma from a biopsy, and was observed for external proceedings without any abnormal findings.

When neurilemmoma was first discovered, there was a mass found from the right back, yet the size was small and MRI

\footnotetext{
- Received: November 16, 2012 - Revised: December 10, 2012

- Accepted: December 18, 2012

Corresponding Author: Sang Gu Lee, MD, PhD

Department of Neurosurgery, Gil Hospital, 1198 block, Guweol-Dong,

Namdong-Gu, Incheon 405-220, Republic of Korea

Tel: +82-32-460-3304, Fax: +82-32-460-3899

Email: samddal@gilhospital.com
}
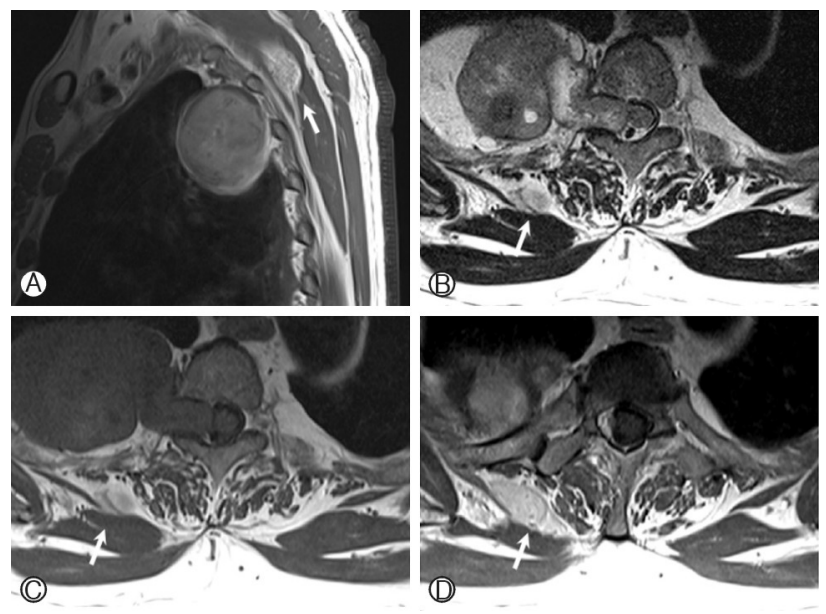

Fig. 1. A. A well defined solid oval mass $(4.9 \times 8.2 \times 5.7 \mathrm{~cm})$ was in right posterior chest wall. B. C. D. Axial T2-weighted (B) and $\mathrm{T} 1$-weighted $(\mathrm{C})$ images show dumbbell shaped portion into the right neural foramen of T3-4, compressing spinal cord to the left side. And post contrast enhancement (D) image shows, heterogeneous tumor mixed with solid and cystic component. And in paraspinal muscle, fusiform fatty mass was seen (white arrow).

showed no hints of invasive remark. The patient visited our hospital for further workup after the size of the mass increased gradually.

Clinically, the oval-shaped mass on the patient's back was visible to the naked eye. The size was approximately $12 \times 7 \times 3$ $\mathrm{cm}$, and there were no other abnormal findings such as change of color, ecchymosis, fossula formation etc.

During a physical exam, there was local heat sense found around the mass, but there were no tenderness, or any neurologic abnormal findings such as motor paralysis or hypoesthesia.

For a detailed examination of the mass, a thoracic MRI was performed. From the result, a mass with an approximate size of $12 \times 7 \times 3 \mathrm{~cm}$ was found within the right paraspinal muscle, 

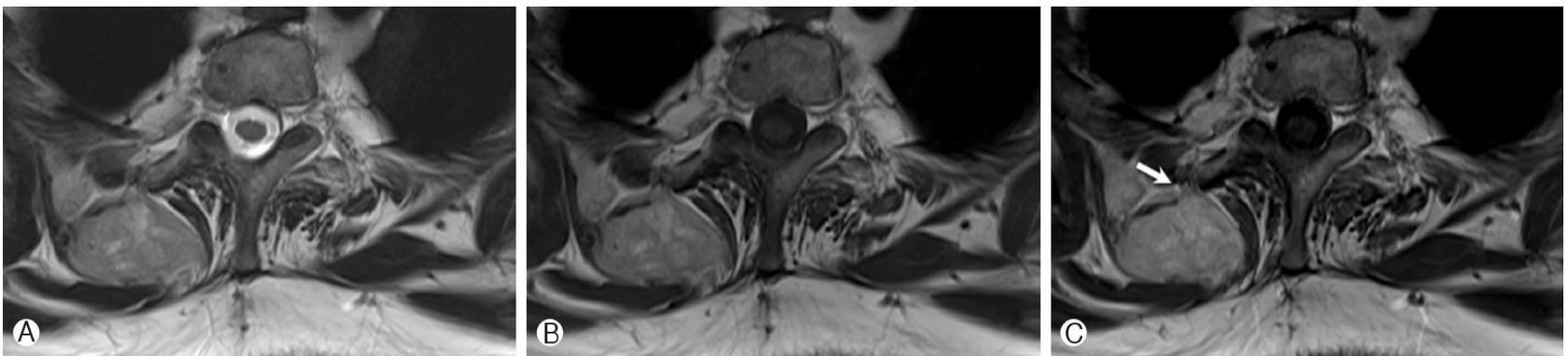

Fig. 2. A, B. At the T3 level, axial T2-weighted (A) and $\mathrm{T} 1$-weighted (B) images show heterogenous high signal intensity soft tissue mass in right paraspinal muscle. C. A post-gadolinium T1-weighted image shows mild septal enhancement within the tumor and an curvilinear signal void lesion, anterior inferior to the tumor, suggestive of vessel sweeping below the tumor ( $C$, white arrow).

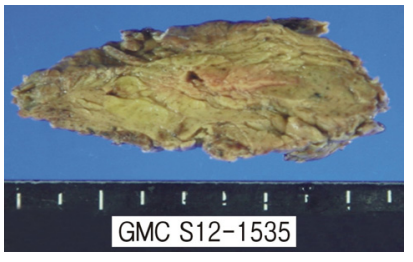

Fig. 3. Gross yellow-brownishpathologic specimen shows yellow-brownish solid fatty tumor with some pinkish pigmentation in the mass.

with a clear margin. It was lobulated, and appeared to be stronglyheterogenous from both T1, T2 myelogram.

In the operating room, the patient was laid on the table in prone position. After the incision, the vertical center from the sixth cervical vertebra to the fourth thoracic vertebra was desquamated to the subcutaneous tissues by skin incision. Then we approached the mass by desquamating along the left border of the vertebra. The mass was yellowish brown, located at the intersection where the connection part of cervical vertebrae and thoracic vertebrae, and surrounded by numerous blood vessels.

Being careful not to injure any neurovascular structures around the mass, a resection of the entire mass was performed, followed by scrupulous hemostasis. There were no neurological abnormalities after the operation.

The mass was $13.6 \times 6.0 \times 2.2 \mathrm{~cm}$ in size, and appeared to be encapsulated with irregular borders of yellow and brown colors. There were no hemorrhage or necrosis, and the crosssection of the mass was observed to be multi-lobulated (Fig. 3). Histologically, it was a typical hibernoma, where polygonal and multivacuolated adipose cells, along with abundant granular cytoplasm, were divided into minute reticulin fiber septum and arranged into a form of lobules (Fig. 4)

Thoracic tomography performed after the operation showed only a small amount of undercurrent body fluid, without other specific findings such as tumor remnants. The operated area did not have peculiar symptoms or infection, and the patient is now outpatient observation after discharge without particular complications. The following hibernoma was accompanied by the history of neurilemmoma, and since several studies
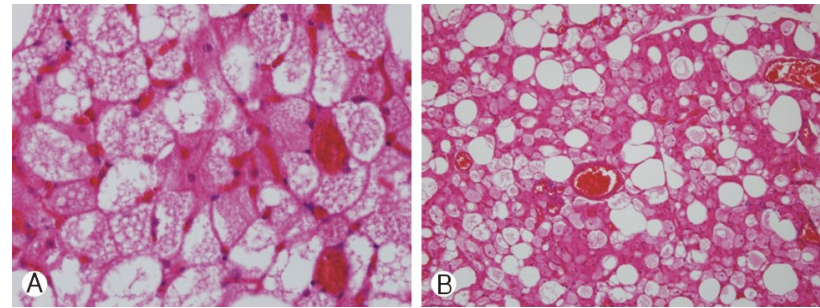

Fig. 4. Histological resection of hibernoma shows inter-lobular connective tissue stroma and typical multilobulated fat tissue with vascular proliferation $(\mathrm{A}: \times 400, \mathrm{~B}: \times 100)$.

in the past reported related chromosome abnormality to these two types of tumor, additional chromosome test was carried out for checkup; the result appeared to be normal without any abnormal findings.

\section{DISCUSSION}

Hibernoma is a benign tumor that originates from remnants of brown fat tissue. Because its cells resembled the cells of hibernating animals, Gery first used the term "Hibernoma" in 1914. From then, hibernoma has been a rare type of tumor, reported by only around 160 cases globally, and 10 cases nationally ${ }^{2,4)}$.

Hibernoma is usually presents without pain, and it gradually grows in size. It commonly develops in one's adolescence or twenties, and slightly appears more in women than in men ${ }^{1)}$. Due to the vascular activity of the mass, the skin that covers the mass may seem warmer than the other parts according to physical exams ${ }^{6}$.

When diagnosing hibernoma, MRI, angiography, and needle biopsy play an important role: it shows lower signal than subcutaneous fat at T1 weighted MRI, increased heterogenous signal in T2 weighted MRI, noticeably increased build-up from Gadolinium imaging ${ }^{1)}$, and a distinguished image from a typical fat mass on fat suppression imaging because of its uncon- 
trolled fat.

MRI can be helpful in checking the size of the mass, vessel conditions, or any permeated parts in terms of radiation beforethe operation. However, it is hard to actually diagnose through radiation results alone, because other tumors including fibroma, neural fibroma, polymorphic lipoma, and positive lipoblastoma display the same findings as hibernoma. At lipoma and hibernoma's MRI, lipoma shows equivalent signal strength while hibernoma's signal is presented with dysplasia on the inner parts due to its vascular fiber structure. Hibernoma is not composed of one particular structure, but a mixture of traditional lipoma and hibernoma component, two complex tumors are integrated as one. The difference of signal strength in MRI is decided by the composition. If the composition of brown fat is higher than the other, it displays lower signal in T1 image and higher signal in T2 image ${ }^{1,8)}$. Because hibernoma shows strengthened vessel structure due to the copious blood distribution,it can be easily distinguished from the mass that has low blood vessel distribution, including simple lipoma, fibroma, neural fibroma and etc, yet it still requires a specialized method to distinguished it from liposarcoma. Knowing the vessel distributionaround the surrounding area can be helpful before planning resection ${ }^{7}$.

Although definite diagnosis before the operation is possible via needle biopsy in most cases, it is recommended to avoid needle biopsy if there are too many vessels formed around the mass, which may result in excessive bleeding ${ }^{9}$.

In this case, the mass was a painless tumor according to the physical exam, only with a slight heat sensation around the mass area. MRI findings showed clear difference from a simple lipoma, which has similar signal strength to subcutaneous fat tissue, and seen from heterogenous aspects and high vessel distribution, the tumor had to be distinguished from low grade liposarcoma and angiolipoma ${ }^{8)}$.

There was a case of malignant hibernoma in the past, but in most of cases, it can be treated sufficiently by resection alone, with few sequelae. However, in terms of abundant vessel distribution of hibernoma, sometimes it can be hard to remove the tumor entirely. Hematoma may result after operation, and resection alone cannot totally eliminate the possibility of malignancy in terms of histology. Therefore, performing a total resection reduces the chance of the recurrence of tumor remnants.

After the operation, extra procedure is required to prevent hemostasis. If needed, angiography should be carried out beforehand. If there is a nutrition vessel found, embolization of the vessel is required to minimize the bleeding that may occur during the actual operation ${ }^{4,5,6}$.

In 1994, Fredrik et al. presented seven case reports in total, including three previous case reports, about chromosome abnormalities occurring in hibernoma patients ${ }^{3)}$. Thus, it is gen- erally thought that hibernoma patients have a structural abnormalityof chromosome 11's long arm, including 11q13. Also, numerous neurilemmoma are discovered in Type 2 neurofibromatosis, caused by a problematic sex chromosome. For the following patient in this case, abnormal sex chromosome were suspected ascause for neurilemmoma and hibernoma.

Actual chromosome test results proved that this patient had a normal karyotype without unusual number of chromosomes or structural disorder.

\section{CONCLUSION}

This case report presents a case of hibernoma that occurred in a patient who previously had an operation for neurilemmoma. It was a rare case of two tumors presenting at the same time, which is hard to find in previous literature. After diagnosis, thepatient was treated with total resection of each tumor, and upon observation showed no post-op complications or sequelae. One should keep in mind that if hibernoma is identified via imaging tests and biopsy in future cases, the tumor can be fully treated by total resection, and that excessive bleeding may occur due to surrounding blood vessels formed anew. Such knowledge and guidelines will benefit in additional chromosome examination of a hibernoma patient, and diagnosis and additional data collection.

\section{REFERENCES}

1. Cook MA, Stern M, de Silva RD: MRI of hibernoma. J Comput Assis Tomogr 20:333-335, 1996

2. Enzinger FM, Weiss SW: Soft tissue tumors. London, CV Mosby: 234-241, 1983

3. Mertens F, Rydholm A, Brosjö O, Willén H, Mitelman F, Mandahl N: Hibernomas are characterized by rearrangements of chromosome bands 11q13-21 Int J Cancer 58:503-505, 1994

4. Furlong MA, Fanburg-Smith JC, Miettinen M: The morphologic spectrum of hibernoma: A clinicopathologic study of 170 cases. Am J Surg Pathol 25:809-814, 2001

5. HA JW, Kim HS, Park JO, Chung JY, Pyo JY: Hibenoma in psoas muscle. J of Korean Spine Surg 13:206-310, 2006

6. Lewandowski PJ, Weiner SD: Hibernoma of the medial thigh. Case report and literature review. Clin Orthop Relat Res 330: 198-201, 1996

7. McLane RC, Meyer LC: Axillary hibernoma: review of the literature with report of a case examined angiographically. Radiology 127:673-674, 1978

8. Munk PL, Lee MJ, Janzen DL, Connell DG, Logan PM, Poon PY, et al: Lipoma and liposarcoma: evaluation using CT and MR imaging. AJR Am J Roentgenol 169:589-594, 1997

9. Rigor VU, Goldstone SE, Jones J, Bernstein R, Gold MS, Weiner S: Hibernoma. A case report and discussion of a rare tumor. Cancer 57:2207-2211, 1986 